\title{
Experimental device-independent tests of classical and quantum dimensions
}

\author{
Johan Ahrens ${ }^{1}$, Piotr Badziạg ${ }^{1}$, Adán Cabello, ${ }^{1,2}$ and Mohamed Bourennane ${ }^{1 \star}$
}

\begin{abstract}
A fundamental resource in any communication and computation task is the amount of information that can be transmitted and processed. The classical information encoded in a set of states is limited by the number of distinguishable states or classical dimension $d_{c}$ of the set. The sets used in quantum communication and information processing contain states that are neither identical nor distinguishable, and the quantum dimension $d_{\mathrm{q}}$ of the set is the dimension of the Hilbert space spanned by these states. An important challenge is to assess the (classical or quantum) dimension of a set of states in a device-independent way, that is, without referring to the internal working of the device generating the states. Here we experimentally test dimension witnesses designed to efficiently determine the minimum dimension of sets of (three or four) photonic states from the correlations originated from measurements on them, and distinguish between classical and quantum sets of states.
\end{abstract}

Classical and quantum dimensions are fundamental quantities in information processing. In particular, the security of many cryptographic schemes ${ }^{1-3}$ crucially relies on the dimensional characteristics of the information carriers. From a fundamental perspective, the difference between classical and quantum dimensions can be used for quantification of the non-classicality of correlations: classical simulation of correlations produced by a quantum system of (quantum) dimension $d_{\mathrm{q}}$ may require a classical system of (classical) dimension $d_{\mathrm{c}} \gg d_{\mathrm{q}}$ (refs 4-6).

The problem of efficiently testing the minimum possible dimension spanned by a set of states has been approached from different theoretical perspectives. The concept of a quantum dimension witness was first introduced for the dimension of the Hilbert space of composite systems tested locally ${ }^{7}$, and then related to the construction of quantum random access codes ${ }^{8}$ and approached from a dynamical viewpoint ${ }^{9}$.

A device-independent approach, that is, without any reference to the internal working of the device generating the states (state preparator) was introduced recently ${ }^{10}$. In this scenario, the measurement device must be trusted. Such trust can be based on, for example, the device successfully passing suitable tests before the test of the state preparator. Moreover, one has to assume that the manufacturers of the state preparator and the measurement device do not conspire against the user. This implies that there are no secret communication channels or preprogrammed correlations between the state preparator and the measurement device.

The state preparation and the tests performed under these premises are shown schematically in Fig. 1. There is a state preparator with $N$ buttons; it emits a particle in a state $\rho_{x}$ (specified by the device's supplier) when button $x \in\{1, \ldots, N\}$ is pressed. For testing, the emitted particles are sent to a measurement

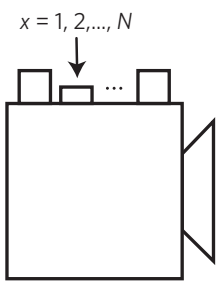

State preparator

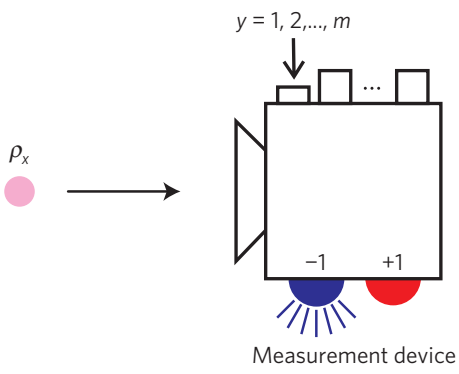

Figure 1 | Device-independent scenario for testing the minimum classical or quantum dimension. A state preparator with $N$ buttons emits a particle in a state $\rho_{x}$ when button $x \in\{1, \ldots, N\}$ is pressed. This particle is sent to a measurement device with $m$ buttons. When button $y \in\{1, \ldots, m\}$ is pressed, the device performs measurement $M_{y}$ on the particle. The measurement produces outcome $b \in\{-1,+1\}$.

device, with $m$ buttons. When button $y \in\{1, \ldots, m\}$ is pressed, the device performs measurement $M_{y}$ on the incoming particle. The measurement produces outcome $b \in\{-1,+1\}$. A complete test consists of many measurements on all of the states. It should yield a probability distribution $P(b \mid x, y)$ for obtaining result $b$ in measurement $y$ on state $\rho_{x}$. Suitable combinations of the experimental probabilities $P(b \mid x, y)$ can then be compared with the theoretical bounds for the values of the corresponding (classical or quantum) dimension witnesses.

For our estimations of the lower bounds for $d_{\mathrm{c}}$ and $d_{\mathrm{q}}$ we use recently proposed dimension witnesses ${ }^{10}$. These witnesses use as primary quantities the expectation values

$$
E_{x y}=P(+1 \mid x, y)-P(-1 \mid x, y)
$$

In our tests we use two combinations of these expectation values called $I_{3}$ and $I_{4}$. The first combination, $I_{3}$, works both as a tight two-dimensional classical witness and a two-dimensional quantum witness. In other words, it allows one to identify sets of states with $d_{\mathrm{c}} \geq 2$ and $d_{\mathrm{q}} \geq 2$. It uses three preparations, which means three potentially different states $(N=3)$ and two dichotomic measurements $(m=2)$. The corresponding inequalities are:

$$
I_{3} \equiv\left|E_{11}+E_{12}+E_{21}-E_{22}-E_{31}\right| \stackrel{\text { bit }}{\leq} \stackrel{\text { qubit }}{\leq} 1+2 \sqrt{2} \stackrel{\text { trit, qutrit }}{\leq} 5
$$

where $\stackrel{\text { bit }}{\leq} 3$ means that no classical system of dimension $d_{\mathrm{c}}=2$ can give a value larger than 3 , and $\underset{\leq}{\text { qubit }} 1+2 \sqrt{2}$ means that no quantum system of dimension $d_{\mathrm{q}}=2$ can give a value larger than $1+2 \sqrt{2} \approx 3.8284$. Finally, $\stackrel{\text { trit, qutrit }}{\leq} 5$ means that no classical system of

${ }^{1}$ Physics Department, Stockholm University, S-10691 Stockholm, Sweden, ${ }^{2}$ Departamento de Física Aplicada II, Universidad de Sevilla, E-41012 Sevilla Spain. *e-mail: boure@fysik.su.se. 


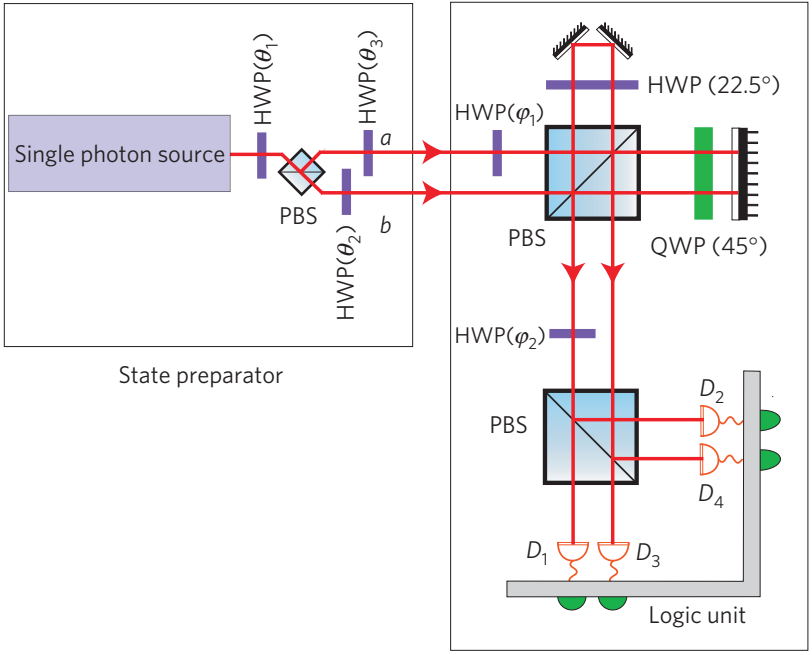

Measurement device

Figure 2 | Experimental set-up for testing classical and quantum dimension witnesses. The state preparator is a single-photon source emitting horizontally polarized photons that, after passing through three half-wave plates suitably oriented at angles $\theta_{i}$ (with $i=1,2,3$ ), are prepared in the required states. Information is encoded in horizontal and vertical polarizations, and in two spatial modes. The probabilities needed for the dimension witnesses are obtained from the number of detections in the detectors $D_{i}$, after properly adjusting the orientations $\varphi_{i}$ (with $i=1,2,3$ ) of the half-wave plates in the measurement device. QWP, quarter-wave plate; PBS, polarizing beam splitter.

dimension $d_{\mathrm{c}}=3$ or quantum system of dimension $d_{\mathrm{q}}=3$ can give a value larger than 5 , which is the algebraic maximum of $I_{3}$.

The second combination, $I_{4}$, tests sets of four states $(N=4)$ and uses three dichotomic measurements $(m=3)$. It represents witnesses represented by the following inequalities:

$$
\begin{aligned}
I_{4} & \equiv\left|E_{11}+E_{12}+E_{13}+E_{21}+E_{22}-E_{23}+E_{31}-E_{32}-E_{41}\right| \\
& \quad \leq 5 \stackrel{\text { bit }}{\leq} 56 \stackrel{\text { qubit }}{\leq} \stackrel{\text { trit }}{\leq} \stackrel{\text { qutrit }}{\leq} 2+\sqrt{13+16 \sqrt{2}} \stackrel{\text { quart,ququart }}{\leq} 9
\end{aligned}
$$

Thus, $I_{4}$ is a dimension witness capable of identifying $d_{\mathrm{c}} \geq 2,3$ and $d_{\mathrm{q}} \geq 2,3$. One may notice that the power of the measurement devices relies to a large extent on the user's degree of control of this device. For instance, with a limited knowledge of what the device is actually measuring, the user obtaining an outcome 5.8 for $I_{4}$ will not know whether the states represent a 'dirty' 3-dim or 4-dim set or a quantum 2-dim set of states. On the other hand, a user who knows that the measurements were confined to a 2-dim Hilbert space can be sure that the tested set of states is genuinely quantum.

The state preparator in Fig. 2 emits photons in which information is encoded in horizontal $(\mathrm{H})$ and vertical $(\mathrm{V})$ polarizations, and in two spatial modes $(a$ and $b)$. We define four basis states: $|0\rangle \equiv|\mathrm{H}, a\rangle,|1\rangle \equiv|\mathrm{V}, a\rangle,|2\rangle \equiv|\mathrm{H}, b\rangle$ and $|3\rangle \equiv|\mathrm{V}, b\rangle$. With these encodings, any qubit state can be represented as $\alpha|\mathrm{H}, a\rangle+\beta|\mathrm{V}, a\rangle$, any qutrit state as $\alpha|\mathrm{H}, a\rangle+\beta|\mathrm{V}, a\rangle+\gamma|\mathrm{H}, b\rangle$ and any ququart state as $\alpha|\mathrm{H}, a\rangle+\beta|\mathrm{V}, a\rangle+\gamma|\mathrm{H}, b\rangle+\delta|\mathrm{V}, b\rangle$.

In our experiments, the photonic states were prepared as follows: a single-photon source (see the state preparator frame in Fig. 2) emitted a horizontally polarized photon. On passing through three suitably oriented half-wave plates $\operatorname{HWP}\left(\theta_{1}\right), \operatorname{HWP}\left(\theta_{2}\right)$ and $\operatorname{HWP}\left(\theta_{3}\right)$, the state of the emitted photon was converted to the required state $|\psi\rangle$

$$
\begin{aligned}
|\psi\rangle= & \sin \left(2 \theta_{1}\right) \cos \left(2 \theta_{2}\right)|\mathrm{H}, a\rangle+\sin \left(2 \theta_{1}\right) \sin \left(2 \theta_{2}\right)|\mathrm{V}, a\rangle \\
& +\cos \left(2 \theta_{1}\right) \cos \left(2 \theta_{3}\right)|\mathrm{H}, b\rangle+\cos \left(2 \theta_{1}\right) \sin \left(2 \theta_{3}\right)|\mathrm{V}, b\rangle
\end{aligned}
$$

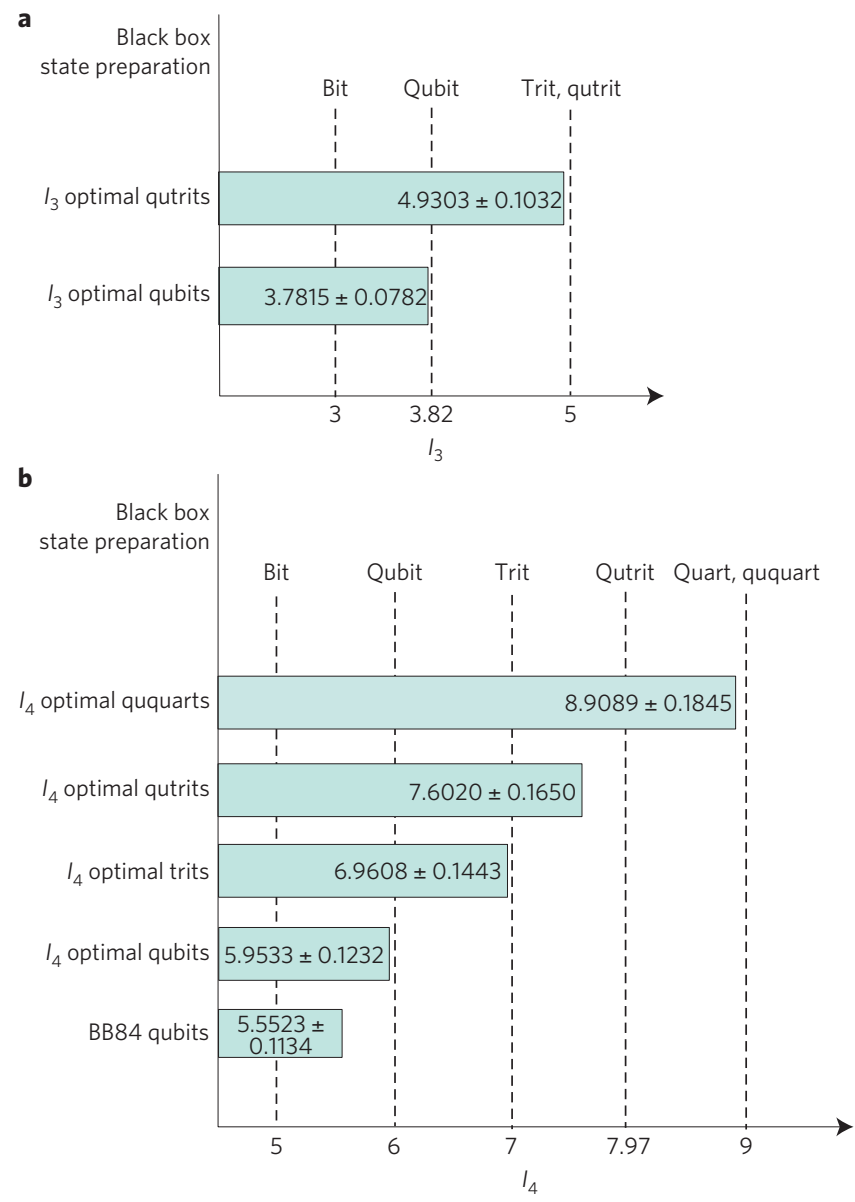

Figure 3 | Experimental results of the dimension witness tests. The vertical dashed line labelled 'bit' represents the maximum value achievable with bits, and similarly for the other vertical dashed lines. 13 optimal qutrits means that the black box emits qutrit states that give the maximum value for $I_{3}$ using qutrits, and similarly for the other preparations. BB84 qubits denotes the states used in standard quantum cryptography.

Thus, by adjusting the HWP orientation angles $\theta_{i}$, we could produce any of the states in the experiment. Classical sets (bits, trits, quarts) consisted of states designed to be perfectly distinguishable or identical.

The measurement device could be set in different ways. For experiments with qubit states, $P(+1 \mid x, y)$ was obtained from the number of detections in $D_{1}$, and $P(-1 \mid x, y)$ was obtained from the number of detections in $D_{3}$. Otherwise for experiments with qutrit states, $P(+1 \mid x, y)$ was obtained from the number of detections in $D_{1}$ and $D_{2}$, and $P(-1 \mid x, y)$ was obtained from the number of detections in $D_{3}$. When the state preparator announced classical sets, the measurement settings became particularly simple and reduced to arranging the detectors so that they clicked on receiving a photon in a particular basis state: $|0\rangle \rightarrow D_{1},|1\rangle \rightarrow D_{3},|2\rangle \rightarrow D_{2}$ and $|3\rangle \rightarrow D_{4}$. The sought for measurement settings were obtained by adjusting the orientations $\varphi_{i}$ of the half-wave plates $\operatorname{HWP}\left(\varphi_{1}\right)$ and $\operatorname{HWP}\left(\varphi_{2}\right)$ (see the measurement device frame in Fig. 2).

Our single-photon source was weak coherent light from a stabilized narrow-bandwidth diode laser emitting at $780 \mathrm{~nm}$ and offering a long coherence length. The laser was attenuated so that the two-photon coincidences were negligible. Our single-photon detectors $\left(D_{i}, i=1,2,3,4\right)$ were silicon avalanche photodiodes with detection efficiency $\eta_{\mathrm{d}}$ and dark counts rate $R_{\mathrm{d}}$. For our detectors these values were around $\eta_{\mathrm{d}} \simeq 0.55$ and $R_{\mathrm{d}} \simeq 400 \mathrm{~Hz}$, but they varied slightly from detector to detector. The overall detection efficiency 
Table 1 | Experimental results of the dimension witness tests.

\begin{tabular}{|c|c|c|c|c|c|c|}
\hline Inequality bound & $I_{\text {th }}$ & $I_{\text {exp }}$ & $I_{\mathrm{exp}}^{b}$ & $\Delta I_{\mathrm{p}}$ & $\Delta I_{\mathrm{d}}$ & $\Delta I_{\mathrm{T}}$ \\
\hline$I_{3}$ (qubit) & 3.8284 & 3.6533 & 3.7815 & 0.0772 & 0.0125 & 0.0782 \\
\hline$I_{3}$ (qutrit) & 5 & 4.7119 & 4.9303 & 0.1021 & 0.0150 & 0.1032 \\
\hline$I_{4}(\mathrm{BB} 84)$ & 5.6503 & 5.5210 & 5.5523 & 0.1126 & 0.0133 & 0.1134 \\
\hline$I_{4}$ (qubit) & 6 & 5.7554 & 5.9533 & 0.1221 & 0.0163 & 0.1232 \\
\hline$I_{4}$ (trit) & 7 & 6.7595 & 6.9608 & 0.1433 & 0.0171 & 0.1443 \\
\hline$I_{4}$ (qutrit) & 7.9689 & 7.2909 & 7.6020 & 0.1596 & 0.0419 & 0.1650 \\
\hline$I_{4}$ (quart) & 9 & 8.5181 & 8.9089 & 0.1833 & 0.0209 & 0.1845 \\
\hline
\end{tabular}

$I_{\text {th }}, I_{\exp }$ and $I_{\exp }^{b}$ represent the theoretical, raw experimental and dark-count-corrected experimental values of the dimension witness bounds, respectively. $\Delta I_{\mathrm{p}}, \Delta I_{\mathrm{d}}$ and $\Delta I_{\mathrm{T}}$ are the errors due to the limited precision of the settings of the polarization components and the imperfections of the polarization splitting, the propagated Poissonian counting statistics of the raw detection events and the total errors, respectively.

$\eta$ of each $D_{i}$ is a product $\eta_{\mathrm{d}} \eta_{\mathrm{c}}$, where $\eta_{\mathrm{c}}$ is the fibre coupling efficiency (with typical maximal value $\eta_{\mathrm{c}} \simeq 0.90$ ). To secure the same overall detection efficiency $\eta$ for all of the detectors in the experiment, we measured $\eta_{\mathrm{d}}$ independently of the fibre coupling for each detector separately and then adjusted the coupling efficiency $\eta_{c}$. This calibration of the overall detection efficiencies included even the mismatches in the transmission and reflection losses in the polarization beam splitters.

The detectors $D_{i}$ produced output transistor-transistor logic signals of $4.1 \mathrm{~V}$ (with duration of $41 \mathrm{~ns}$ ). The dead time of the detectors was $50 \mathrm{~ns}$. All single counts were registered using multichannel coincidence logic with a time window of $1.7 \mathrm{~ns}$.

The first two experiments were $I_{3}$ tests. The first goal was to obtain the maximum qubit violation of the bit bound $I_{3}$ (bit) $=3$. For this purpose, we prepared $N=3$ qubit states and performed $m=2$ dichotomic measurements intended to maximize the value of $I_{3}$. The optimal states and measurements for all experiments are described in the Methods.

The goal of the second experiment was to obtain the maximum qutrit violation of the qubit bound $I_{3}$ (qubit) $\approx 3.8284$. For this, we prepared $N=3$ qutrit states and performed $m=2$ dichotomic measurements. The results yielded $I_{3}$ very close to the algebraic bound $I_{3}=5$.

The remaining experiments were $I_{4}$ tests. The goal of the third experiment was to obtain the maximum qubit violation of the bit bound $I_{4}($ bit $)=5$. We prepared $N=4$ qubit states and performed $m=3$ dichotomic measurements that maximize $I_{4}$. The goal of the fourth experiment was to obtain the maximum trit violation of the qubit bound $I_{4}$ (qubit) $=6$. We prepared $N=4$ trit states and performed $m=3$ dichotomic measurements that maximize $I_{4}$. The goal of the fifth experiment was to obtain the maximum qutrit violation of the trit bound $I_{4}$ (trit) $=7$. We prepared $N=4$ qutrit states and performed $m=3$ dichotomic measurements that maximize $I_{4}$. The sixth experiment was an $I_{4}$ test on quarts. The goal was to obtain the maximum quart violation of the qutrit bound $I_{4}$ (qutrit) $=7.96887$. For this, we prepared $N=4$ quart states and performed $m=3$ dichotomic measurements. As in the corresponding $I_{3}$ test, the results gave $I_{4}$ very close to the algebraic bound $I_{4}=9$.

The states that saturate the witnesses boundaries may not be the most valuable for information processing. It is therefore interesting to test the dimension for the set of states that are actually used for information processing purposes. For quantum cryptography, a valuable set of states consists of four pairwise orthogonal and pairwise unbiased qubit states, such as $\left|\psi_{1}\right\rangle=|0\rangle$, $\left|\psi_{2}\right\rangle=(1 / \sqrt{2})(|0\rangle+|1\rangle),\left|\psi_{3}\right\rangle=|1\rangle$ and $\left|\psi_{4}\right\rangle=(1 / \sqrt{2})(|0\rangle-|1\rangle)$ (ref. 11). Thus, in the seventh experiment we tested the violation of the bit bound $I_{4}\left(d_{\mathrm{c}}=2\right)=5$ by the four cryptographic states. For this, we performed the measurements maximizing the value of $I_{4}$ for these states (see Methods for details). Theoretically, this maximal value is $I_{4}(\mathrm{BB} 84)=\sqrt{2}+2+\sqrt{5} \approx 5.6506$, that is, a value that clearly exceeds the bit bound $I_{4}\left(d_{c}=2\right)=5$.

All of our experimental results are summarized in Fig. 3. The obtained experimental values are in a very good agreement with the theoretical predictions. This clearly demonstrates that we are able to determine the minimum dimension of a supplied set of states. The main sources of systematic errors were due to imperfection of the optical interferometer involved in the measurements, the non-perfect overlapping of the light modes and the polarization components. The errors were deduced from propagated Poissonian counting statistics of the raw detection events, the limited precision of the settings of the polarization components (HWP plates) and the imperfection of the polarizing beam splitters. The number of detected single photons was about $1.5 \times 10^{5} \mathrm{~s}^{-1}$ and the coincidence to single ratio was less than $2 \times 10^{-4}$. The measurement time for each experiment was $30 \mathrm{~s}$. All of the results and their corresponding errors are listed in Table 1.

We have experimentally determined lower bounds for the dimension of several ensembles of physical systems in a deviceindependent way. For the tests, we used classical and quantum dimension witnesses recently derived ${ }^{10}$. The witnesses used optimal measurements and were applied to sets of photonic bits, qubits, trits, qutrits and quarts in optimal states for maximal violation of the corresponding dimension witnesses. In addition, we applied a dimension witness $I_{4}$ to the four qubit states used in standard quantum cryptography.

Our results demonstrate how dimension witnesses can be used to test classical and quantum dimensions of sets of physical states generated in externally supplied, potentially defective devices and how one can distinguish between classical and quantum sets of states of a given dimension. A very good agreement between the experimental results and the theoretical predictions makes us believe that the method can be extended to more complex witnesses and to tests of systems claiming to span higher dimensions.

\section{Methods}

Maximum qubit violation of the bit bound of $I_{3}$. To design $N=3$ qubit states and $m=2$ dichotomic measurements that maximize the value of $I_{3}$, we consider the two dichotomic measurements

$$
M_{y}=\mathbb{1}-2\left|m_{y}\right\rangle\left\langle m_{y}\right|
$$

where $\mathbb{1}$ denotes the identity matrix and

$$
\left|m_{1,2}\right\rangle=\cos \left(\frac{\alpha}{2}\right)|0\rangle \mp \sin \left(\frac{\alpha}{2}\right)|1\rangle
$$


The three prepared states can be chosen as pure states $\rho_{x}=\left|\psi_{x}\right\rangle\left\langle\psi_{x}\right|$, where

$$
\left|\psi_{3}\right\rangle=\cos \left(\frac{\alpha}{2}\right)|0\rangle-\sin \left(\frac{\alpha}{2}\right)|1\rangle=\left|m_{1}\right\rangle
$$

The optimization of the set-up can thus be reduced to finding the maximum of the sum of the larger eigenvalues of $M_{1}+M_{2}$ and $M_{1}-M_{2}$. This fixes parameter $\alpha$ to $\alpha_{\text {opt }}=\pi / 4$ with the following result:

$$
\left|m_{1,2}\right\rangle=\frac{\sqrt{2+\sqrt{2}}}{2}|0\rangle \mp \frac{\sqrt{2-\sqrt{2}}}{2}|1\rangle
$$

States $\left|\psi_{1}\right\rangle$ and $\left|\psi_{2}\right\rangle$ are then the corresponding eigenvectors, $\left|\psi_{1}\right\rangle=|1\rangle$ and $\left|\psi_{2}\right\rangle=(1 / \sqrt{2})(|0\rangle+|1\rangle)$

Maximum qutrit violation of the qubit bound of $I_{3}$. To reach the algebraic bound $I_{3}=5$ with qutrits, we used

$$
\begin{aligned}
& M_{1}=|0\rangle\langle 0|-| 1\rangle\langle 1|+| 2\rangle\langle 2| \\
& M_{2}=|0\rangle\langle 0|+| 1\rangle\langle 1|-| 2\rangle\langle 2|
\end{aligned}
$$

and the states $\left|\psi_{1}\right\rangle=|0\rangle,\left|\psi_{2}\right\rangle=|2\rangle$ and $\left|\psi_{3}\right\rangle=|1\rangle$.

Maximum qubit violation of the bit bound of $\boldsymbol{I}_{\mathbf{4}}$. To determine the maximum qubit violation of $I_{4}$, we generalize the procedure used for $I_{3}$. We consider three measurements $M_{y}(y=1,2,3)$, with $\left|m_{1}\right\rangle$ and $\left|m_{2}\right\rangle$ defined in (1) and (2). State $\left|m_{3}\right\rangle$ is arbitrary. The optimization of the set-up is now reduced to maximizing the sum of the largest eigenvalues of $M_{1}-M_{2}, M_{1}+M_{2}+M_{3}$ and $M_{1}+M_{2}-M_{3}$. It brings the optimal value of $\alpha$ to $\alpha_{\mathrm{opt}}=\pi / 6$ and $\left|m_{3}\right\rangle=(1 / \sqrt{2})(|0\rangle-|1\rangle)$. The corresponding states are then the eigenvectors belonging to the maximal eigenvalues of $M_{1}+M_{2}+M_{3}, M_{1}+M_{2}-M_{3}, M_{1}-M_{2}$ and $-M_{1}$, that is,

$$
\begin{gathered}
\left|\psi_{1}\right\rangle=(2+\sqrt{3})|0\rangle+|1\rangle \\
\left|\psi_{2}\right\rangle=(2+\sqrt{3})|0\rangle-|1\rangle \\
\left|\psi_{3}\right\rangle=|0\rangle+|1\rangle \\
\left|\psi_{4}\right\rangle=\left|m_{1}\right\rangle
\end{gathered}
$$

Maximum trit violation of the qubit bound of $\boldsymbol{I}_{\mathbf{4}}$. The optimal preparations are $\left|\psi_{1}\right\rangle=\left|\psi_{2}\right\rangle=|0\rangle,\left|\psi_{3}\right\rangle=|2\rangle$ and $\left|\psi_{4}\right\rangle=|1\rangle$, and the optimal measurements are

$$
\begin{aligned}
& M_{1}=|0\rangle\langle 0|-| 1\rangle\langle 1|+| 2\rangle\langle 2| \\
& M_{2}=|0\rangle\langle 0|+| 1\rangle\langle 1|-| 2\rangle\langle 2| \\
& M_{3}=|0\rangle\langle 0|-| 1\rangle\langle 1|-| 2\rangle\langle 2|
\end{aligned}
$$

Maximum qutrit violation of the trit bound of $\boldsymbol{I}_{\mathbf{4}}$. The optimal measurements correspond to the observables of the form (1) with

$$
\left|m_{1,2}\right\rangle=\cos \left(\frac{\alpha}{2}\right)|1\rangle \pm \sin \left(\frac{\alpha}{2}\right)|2\rangle
$$

The optimization proceeds as for qubits, but the algebra is more involved. We obtain

$$
\begin{gathered}
\left|m_{3}\right\rangle=\frac{1}{\sqrt{2}}(|0\rangle+|2\rangle) \\
\left|\psi_{4}\right\rangle=\left|m_{1}\right\rangle \\
\left|\psi_{3}\right\rangle=\frac{1}{\sqrt{2}}(|1\rangle-|2\rangle)
\end{gathered}
$$

and the (unnormalized) $\left|\psi_{2}\right\rangle$ and $\left|\psi_{1}\right\rangle$

$$
\left|\psi_{2,1}\right\rangle=|0\rangle \pm\left[1-\cos \alpha-\sqrt{1+(1-\cos \alpha)^{2}}\right]|2\rangle
$$

The optimal value of $\cos \alpha$ is now $\cos \alpha_{0}=(1 / 2)(1-\sqrt{2}+\sqrt{2 \sqrt{2}-1})=0.4689$. It gives $I_{4}\left(d_{\mathrm{q}}=3\right)=2+\sqrt{13+16 \sqrt{2}}=7.9688$.

Maximum quart violation of the qutrit bound of $\boldsymbol{I}_{\mathbf{4}}$. The optimal preparations are $\left|\psi_{1}\right\rangle=|0\rangle,\left|\psi_{2}\right\rangle=|2\rangle,\left|\psi_{3}\right\rangle=|1\rangle$ and $\left|\psi_{4}\right\rangle=|3\rangle$, and the optimal measurements are

$$
\begin{aligned}
& M_{1}=|0\rangle\langle 0|+| 1\rangle\langle 1|+| 2\rangle\langle 2|-| 3\rangle\langle 3| \\
& M_{2}=|0\rangle\langle 0|-| 1\rangle\langle 1|+| 2\rangle\langle 2|-| 3\rangle\langle 3| \\
& M_{3}=|0\rangle\langle 0|+| 1\rangle\langle 1|-| 2\rangle\langle 2|-| 3\rangle\langle 3|
\end{aligned}
$$

Violation of the bit bound of $I_{4}$ with cryptographic states. The measurement settings maximizing the value of $I_{4}$ for the standard cryptographic states are specified by the vectors

$$
\begin{gathered}
\left|m_{1}\right\rangle=\left|\psi_{4}\right\rangle \\
\left|m_{2}\right\rangle=(c \sqrt{1-p}-s \sqrt{p})|0\rangle+(c \sqrt{p}+s \sqrt{1-p})|1\rangle \\
\left|m_{3}\right\rangle=\frac{c-s}{\sqrt{2}}|0\rangle+\frac{c+s}{\sqrt{2}}|1\rangle
\end{gathered}
$$

where $c=\cos (\pi / 8), s=\sin (\pi / 8)$ and $p=(1 / 2)(1+3 / \sqrt{10})$. These measurement settings give $I_{4}(\mathrm{BB} 84)=\sqrt{2}+2+\sqrt{5} \approx 5.6506$.

Received 4 March 2012; accepted 3 May 2012; published online 10 June 2012

\section{References}

1. Acín, A., Gisin, N. \& Masanes, L. From Bell's theorem to secure quantum key distribution. Phys. Rev. Lett. 97, 120405 (2006).

2. Pawłowski, M. \& Brunner, N. Semi-device-independent security of one-way quantum key distribution. Phys. Rev. A 84, 010302 (2011).

3. Li, H-W. et al. Semi-device-independent random-number expansion without entanglement. Phys. Rev. A 84, 034301 (2011).

4. Kleinmann, M., Gühne, O., Portillo, J. R., Larsson, J- $\AA$ \& Cabello, A. Memory cost of quantum contextuality. New J. Phys. 13, 113011 (2011).

5. Cabello, A. \& Joosten, J. J. in Unconventional Computation (eds Calude, C. S., Kari, J., Petre, I. \& Rozenberg, G.) 64-76 (Lecture Notes in Computer Science 6714, Springer, 2011).

6. Cabello, A. in A Computable Universe (ed. Zenil, H.) (World Scientific, 2012).

7. Brunner, N. et al. Testing the dimension of Hilbert spaces. Phys. Rev. Lett. 100, 210503 (2008).

8. Wehner, S., Christandl, M. \& Doherty, A. C. Lower bound on the dimension of a quantum system given measured data. Phys. Rev. A 78, 062112 (2008).

9. Wolf, M. M. \& Pérez-García, D. Assessing quantum dimensionality from observable dynamics. Phys. Rev. Lett. 102, 190504 (2009).

10. Gallego, R., Brunner, N., Hadley, C. \& Acín, A. Device-independent tests of classical and quantum dimensions. Phys. Rev. Lett. 105, 230501 (2010).

11. Bennett, C. H. \& Brassard, G. Proc. IEEE International Conference on Computers, Systems, and Signal Processing, Bangalore, India 175-179 (IEEE, 1984).

\section{Acknowledgements}

The authors thank A. Acín, E. Amselem and R. Gallego for stimulating discussions. This work was supported by the Swedish Research Council (VR), the Linnaeus Center of Excellence ADOPT, the MICINN Project No. FIS2008-05596 and the Wenner-Gren Foundation.

\section{Author contributions}

J.A. carried out the experiments under M.B.'s supervision. P.B., A.C. and M.B. jointly conceived the experiments. All authors analysed the data and wrote the manuscript.

\section{Additional information}

The authors declare no competing financial interests. Reprints and permissions information is available online at www.nature.com/reprints. Correspondence and requests for materials should be addressed to M.B. 